Sociologie et sociétés

\title{
Les voies sociales de la consécration musicale
}

Inégalités instrumentales de reconnaissance et gestion de

l'insuccès en musique classique

\section{The social pathways of musical acclaim}

\section{Inequalities in instrument recognition and management of failure in classical music}

\section{Adrien Pégourdie}

Volume 47, numéro 2, automne 2015

Trajectoires de consécration et transformations des champs artistiques

Paths to recognition and transformations of artistic fields

URI : https://id.erudit.org/iderudit/1036343ar

DOI : https://doi.org/10.7202/1036343ar

Aller au sommaire du numéro

Éditeur(s)

Les Presses de l’Université de Montréal

ISSN

0038-030X (imprimé)

1492-1375 (numérique)

Découvrir la revue

Citer cet article

Pégourdie, A. (2015). Les voies sociales de la consécration musicale : inégalités instrumentales de reconnaissance et gestion de l'insuccès en musique classique. Sociologie et sociétés, 47(2), 137-161.

https://doi.org/10.7202/1036343ar

\section{Résumé de l'article}

Cet article analyse les déterminants sociaux de l'accès à la reconnaissance artistique en musique classique. Récusant les modèles théoriques naturalistes centrés sur le talent comme principe explicatif ultime de la réussite des carrières artistiques, il s'attache à mettre en évidence les conditions sociales de la consécration musicale. Ainsi, le processus de reconnaissance est étudié à travers les inégalités instrumentales qu'il suscite, lesquelles sont étroitement liées à des différenciations sociales. Après avoir mis au jour ces inégalités, qui tendent à consacrer prioritairement des interprètes d'instruments socialement sélectifs, on s'intéresse aux façons de surmonter l'absence de consécration pour des interprètes des instruments plus ouverts socialement qui sont, le plus souvent, poussés vers l'enseignement. L'étude des trajectoires de musiciens révèle ainsi les dynamiques sociales auxquelles elles sont soumises, de l'origine sociale dont dépend notamment la familiarisation précoce avec les principes du champ de la musique classique aux inégalités entre les sexes qui orientent les manières de « se faire à sa position ». 


\section{Les voies sociales de la consécration musicale \\ Inégalités instrumentales de reconnaissance et gestion de l'insuccès en musique classique'}

\section{ADRIEN PÉGOURDIE}

Docteur en sociologie au GRESCO (EA 3815),

Université de Limoges

Adresse: 52, rue Edmond About, 87000 Limoges

Courriel: a.pegourdie@numericable.com

\section{$\mathrm{L}$} 'APPRÉHENSION DES PRINCIPES D'ACCÈS À LA RECONNAISSANCE, et plus largement des mécanismes de la structuration des hiérarchies et des classements artistiques, constitue une problématique centrale pour tout sociologue étudiant les arts. Les analyses des déterminants de la consécration artistique ont donné lieu à plusieurs interprétations sociologiques basées sur des conceptions théoriques opposées, que nous évoquerons ici en prenant le domaine précis des études consacrées à la musique. Pour Pierre-Michel Menger (2009), qui s'inspire de la sociologie américaine des carrières artistiques, la reconnaissance musicale est associée à la réussite professionnelle (que ce soit par le succès commercial et/ou le succès d'estime) et provient d'inégalités initiales minimes de «talent» (Rosen, 1981), amplifiées de manière exponentielle au cours des carrières par la loi des différences cumulatives (Merton, 1968). Si l'influence d'autres facteurs est soulignée, il n'en demeure pas moins que, dans ce modèle, le déterminant premier des conditions de la consécration artistique repose sur le «talent» (Menger, 2010). Un tel présupposé soulève une limite quasi indépassable: celle de l'appréhension sociologique du talent. En effet, faute d'un étalon universel et unanimement reconnu

1. Je remercie Wenceslas Lizé et Manuel Schotté qui ont lu et commenté les différentes versions de cet article. 
qui permettrait de déterminer des hiérarchies « objectives» de l'interprétation ou de la création musicale, comme peut l'être le chronomètre dans le cas de la course à pied (Schotté, 2012), Menger (2002a) reconnaît lui-même les limites des tentatives d'objectivation et de quantification du «talent» ou du «génie» artistique. En ce sens, le modèle qu'il propose s'avère sociologiquement incomplet puisqu'il fait jouer à une propriété, qu'il ne parvient pas à définir ni à objectiver, une place centrale dans la reconnaissance artistique (Godechot, 2010), et qu'il réactive par ce biais des principes d'explication naturalistes (Schotté, 2013), incompatibles avec l'impératif disciplinaire qui impose leur mise à distance (Durkheim, 1988).

Cette critique ne signifie pas que l'on balaie toute référence au «talent» dans les champs artistiques. Néanmoins, plutôt que de tenter de déterminer l'existence ou non d'un «talent», ou une vaine comparaison du niveau du «talent» de chaque instrumentiste, c'est plutôt le processus par lequel un «talent» est reconnu à un musicien et par lequel le musicien se reconnaît du «talent» qu'il faut interroger. Il en est, en fait, de la notion de talent comme de la notion de charisme conceptualisée par Max Weber (1995). Le talent, comme le charisme, relève d'une croyance partagée, par celui qui le possède comme par ceux qui le reconnaissent, en l'exceptionnalité des qualités d'un individu. Il constitue une propriété relationnelle qui ne trouve à s'activer que lorsqu'elle est reconnue par des agents extérieurs à l'individu. Ce qui compte alors n'est pas la validité « réelle» du talent ou du charisme, c'est le procédé par lequel s'instaure la croyance collective en cette qualité. En ce sens, les analyses constructivistes de la consécration musicale semblent plus riches sociologiquement que le modèle économiciste aux ferments naturalistes. Défendant le principe de la construction sociale du «génie», ces travaux (Elias, 1991; DeNora, 1995) proposent de penser les processus sociaux et historiques présidant à l'apparition et la reconnaissance du «talent». Ces analyses ne sauraient toutefois tomber dans une approche strictement relativiste niant totalement les différentes capacités individuelles. Elles restituent les conditions sociales d'activation de qualités rares qui conduisent à l'émergence du «talent» et à la consécration musicale de compositeurs parmi les plus salués (Mozart pour Elias et Beethoven pour DeNora). Nous nous inscrirons dans cet article dans ce même paradigme constructiviste. Il ne s'agit pas de réfuter les compétences propres aux musiciens, mais plutôt de montrer comment les dynamiques sociales demeurent centrales dans le déroulement des carrières et dans les processus de consécration artistique des instrumentistes classiques ${ }^{2}$.

Si la fécondité sociologique des travaux d'Elias et DeNora ne saurait être remise en question, ces derniers ne répondent toutefois pas totalement à la problématique de la reconnaissance musicale. Centrés sur les compositeurs appartenant au «canon musical», ceux-ci éclairent sur les formes les plus «ultimes» et pérennes de la reconnaissance appréhendée sous l'angle de l'accès aux positions de pouvoir spirituel. Or la

2. En cela, nous adoptons un modèle d'analyse similaire à celui que Manuel Schotté (2012) utilise pour décrire les trajectoires sportives des athlètes marocains. 
consécration peut revêtir des aspects moins spirituels et plus temporels (Weber, 1995) et des formes moins standardisées et plus «modestes» caractérisées par l'accès temporaire aux positions dominantes du champ. En outre cette centration sur les compositeurs les plus consacrés, et leur traitement en étude de cas, contribuent à une individualisation du processus de reconnaissance musicale.

L'étude des modalités de la consécration musicale traitées dans cet article propose une focale d'analyse différente. Plutôt que de se centrer sur une sociologie des agents relatant les trajectoires exceptionnelles de consécration des "génies», voire leur capacité de subversion sur la structure d'un champ artistique (Bourdieu, 2013), l'analyse se concentre sur les logiques d'accès aux formes les plus routinisées de reconnaissance des instrumentistes classiques français et donc sur la structure du champ français de la musique classique. L'étude de ces logiques de consécration est appréhendée sous l'angle des disparités entre les instruments. Il s'agit de souligner les inégalités instrumentales dans l'accès aux positions dominantes du champ, de montrer combien ces inégalités instrumentales épousent la division sociale de la pratique instrumentale mise en évidence par des enquêtes préalables sur la même population (Ravet, 2003 ; Coulangeon, 2004 ; Lehmann, 2005), et donc de nuancer l'autonomie du champ de la musique classique. Cette focalisation sur les inégalités instrumentales des modes d'accès à la reconnaissance, qui tend à révéler l'homologie structurale entre la hiérarchisation du champ de la musique classique et la hiérarchisation sociale des instruments, permet alors une mise à distance de la question de l'existence du «talent » et une appréhension sociale des processus de consécration musicale.

La première partie de cet article est consacrée à l'étude de l'interdépendance entre la division sociale du travail instrumental et les probabilités d'accès aux formes les plus routinisées de la reconnaissance en musique classique. Il s'agit de montrer combien les possibilités d'atteindre les positions consacrées sont inégales selon l'instrument de musique et combien ces inégalités sont homologues de la hiérarchisation sociale des instruments. En d'autres termes, plus un instrument est caractérisé par un recrutement social élevé de ses interprètes, plus ces derniers ont des probabilités accrues d'accès à la reconnaissance artistique. La seconde partie de cet article se focalise sur l'une des conséquences de l'homologie structurale entre la hiérarchisation du champ de la musique classique et la hiérarchisation sociale des instruments. En se penchant sur l'analyse détaillée des trajectoires sociales et professionnelles de deux interprètes à la formation d'excellence mais jouant d'un instrument aux faibles possibilités d'accès à la reconnaissance artistique, ce sont les moyens de surmonter l'absence de consécration et de se faire à une position moyenne qui sont évoqués. 


\section{Méthodologie de l'enquête}

Ces analyses s'appuient sur un travail de thèse consacrée aux trajectoires professionnelles d'instrumentistes classiques (Pégourdie, 2013). Les matériaux mobilisés dans la première partie de cet article sont issus de données nationales relatives aux instrumentistes classiques français. À ce titre, ont été analysées et retraitées des enquêtes du Département des études, de la prospective et des statistiques (DEPS) du ministère de la Culture et de la Communication, et des recherches institutionnelles commanditées par l'Association française des orchestres (AFO), les conservatoires nationaux supérieurs de musique (CNSM) de Paris et de Lyon et d'autres organismes de formation musicale. La deuxième partie de l'article se base sur des matériaux recueillis auprès de l'échantillon spécifiquement enquêté: des instrumentistes classiques employés dans une capitale régionale. Cette étude prend pour terrain quatre institutions musicales: un conservatoire à rayonnement régional (CRR), un conservatoire à rayonnement municipal (CRC), un orchestre symphonique et lyrique de dimension nationale et un ensemble baroque de renommée internationale. Sur ces terrains, ont été menés 65 entretiens semi-directifs auprès des instrumentistes et de quelques responsables administratifs, une vingtaine d'observations des cours, des répétitions et des concerts. À cela, s'ajoute la passation d'un questionnaire auprès des 117 musiciens qui constituent l'échantillon avec un taux de réponse de $75,2 \%$.

\section{L'INTERDÉPENDANCE ENTRE LES HIÉRARCHIES SOCIALES ET MUSICALES}

Analyser les modalités sociales de la consécration des instrumentistes classiques français nécessite, en premier lieu, de préciser à quoi renvoient les formes routinisées de la reconnaissance musicale. Celles-ci correspondent à l'accès aux positions dominantes du champ de la musique classique, définition qui est un moyen de les objectiver car ces positions dominantes coïncident avec deux statuts professionnels particuliers: les musiciens indépendants et les permanents d'orchestre. Ces positions se caractérisent par le volume important de capital propre au champ de la musique classique 3 nécessaire à leur occupation: possession des titres de formation musicale les plus consacrés (diplômes des CNSM de Paris ou de Lyon ou de leurs équivalents étrangers), réussite à des concours (concours internationaux et professionnels), dimension internationale de la carrière, montant des cachets ou des rémunérations, etc. ${ }^{4}$ En outre, leurs modes d'obtention, détaillés plus loin, impliquent une reconnaissance par le groupe des pairs et/ou les «intermédiaires du travail artistique» (Lizé, Naudier et Roueff, 2011) de la qualité instrumentale des interprètes. En ce sens, étudier l'accès aux positions domi-

3. Sur la notion de capital spécifique dans les différents champs, voir Gérard Mauger (2006).

4. Pour une description plus détaillée de la constitution et de la caractérisation du champ de la musique classique, voir Adrien Pégourdie (2013). 
nantes paraît idoine pour saisir les principes de la consécration musicale et appréhender les processus sociaux qui la sous-tendent.

\section{Inégalités instrumentales d'accès aux positions dominantes ${ }^{5}$}

Le statut de musicien indépendant recouvre les instrumentistes classiques rémunérés exclusivement aux cachets sans recours aux droits de l'intermittence. Il est réservé aux instrumentistes les plus reconnus et les plus sollicités nationalement et internationalement, nommés également "grands solistes» ou "virtuoses», qui tirent leurs ressources financières du cumul de leurs importants cachets dans diverses institutions musicales. Pour atteindre ce statut particulièrement valorisé en musique classique et réservé à une minorité (difficile à dénombrer du fait de la nature internationale de l'activité et du caractère indépendant du travail), les musiciens indépendants ont passé avec succès, et cela dès leur période de formation, de multiples processus de sélection attestant leur «excellence instrumentale» — précocité dans la maîtrise technique de l'instrument, concours d'entrée dans les conservatoires les plus prestigieux, apprentissage auprès des «maîtres» les plus renommés, concours internationaux, etc. (Wagner, 2004). Ces processus de sélection, qui sont autant des moments d'élection que des phases d'élimination, s'apparentent alors à des rites d'institution, qui affirment le prestige de cette position (Bourdieu, 1982) en restreignant les aspirations de la majorité et en consacrant symboliquement un petit nombre de privilégiés.

Les conditions d'obtention du statut de musicien indépendant ne sauraient toutefois se réduire à la révélation du «talent» ou de la «virtuosité instrumentale» des interprètes. L'instrument de musique en est également un déterminant essentiel, les chances d'accès à ce statut étant largement disparates selon les instruments. Devenir musicien indépendant impliquant de se produire régulièrement en soliste, l'accès à ce statut dépend des potentialités de production soliste de chaque instrument, potentialités liées à l'offre de répertoire par instrument. Ainsi, plus le répertoire soliste d'un instrument est développé autant «quantitativement» que «qualitativement», c'est-àdire à la fois par le nombre des œuvres que par la réputation de leurs compositeurs, plus les occasions de se produire sont potentiellement élevées. Le développement et la reconnaissance des répertoires des instruments à cordes (Lehmann, 2005) favorisent alors leurs interprètes. Le nombre des musiciens indépendants en piano, violon ou violoncelle est ainsi plus important que dans les autres instruments, loin de posséder le même répertoire soliste.

La liste des interprètes solistes employés par l'Orchestre de Paris lors de la saison 2011/2012 souligne ce constat. Parmi les différents programmes présentés par cet ensemble ayant nécessité l'engagement d'un ou de plusieurs solistes, on dénombre

5. Certains éléments présentés dans cette partie ont été évoqués dans un autre article (Pégourdie, 2015) consacré à la division sociale du travail instrumental sur tous les emplois de la musique classique, y compris l'intermittence et l'enseignement. La cohérence de la démonstration de cet article me conduit à les présenter également ici. 
21 pianistes, 15 violonistes, 4 violoncellistes, 2 clarinettistes et 1 harpiste ${ }^{6}$. L'inégalité et la segmentation de cette distribution révèlent l'avantage des interprètes des instruments à cordes, et plus particulièrement du piano et du violon, dans l'obtention des prestations solistes et donc l'adoption du statut de musicien indépendant.

Cette situation privilégiée des instrumentistes à cordes s'observe également pour l'obtention du statut de permanent d'orchestre. Celui-ci renvoie aux musiciens salariés à temps plein dans un orchestre symphonique, bénéficiant d'un contrat à durée indéterminée ou d'un contrat d'une durée de trois ans renouvelable sans limitation. Ces musiciens sont recrutés à la suite d'un concours particulièrement exigeant. Jusqu'à 150 candidats, en fonction des instruments et des orchestres, peuvent ainsi se présenter pour un ou deux postes ouverts (Ravet, 2006).

L'accès à ce statut révèle également des inégalités instrumentales. La première tient dans le fait que tous les instruments ne sont pas représentés dans l'orchestre symphonique. Intégrer un orchestre nécessite ainsi de jouer d'un instrument appartenant au socle commun invariant de l'orchestre symphonique: l'instrumentarium. Celui-ci est constitué de quatre instruments de la famille des cordes (violon, alto, violoncelle, contrebasse), de quatre instruments de la famille des bois (flûte, hautbois, clarinette, basson), de quatre instruments de la famille des cuivres (cor, trompette, trombone, tuba) et de percussions (timbales et autres). À cette base, peuvent ponctuellement s'ajouter, selon les œuvres présentées, une harpe et/ou un clavier (piano ou orgue) et/ ou épisodiquement d'autres instruments. Plusieurs instrumentistes sont donc absents des effectifs de permanents d'orchestre, tels les saxophonistes. Leurs interprètes sont mécaniquement privés d'accès à ce statut et voient leurs possibles professionnels dans l'interprétation largement amputés. Pour les harpistes, les pianistes et les organistes, la situation n'est pas non plus très favorable. N'étant que ponctuellement sollicités, leurs effectifs dans les orchestres sont relativement limités, comme nous le verrons plus loin.

D'autres inégalités instrumentales s'ajoutent à l'appartenance ou non à l'instrumentarium. La répartition instrumentale des effectifs nationaux des permanents d'orchestre se révèle ainsi pour le moins disparate. Le recensement des instrumentistes des 29 orchestres permanents affiliés à l'Association française des orchestres (AFO) $(\mathrm{n}=2003)^{7}$ souligne que les quatre instruments de la famille des cordes (violon, alto, violoncelle, contrebasse) constituent plus de la moitié $(62,7 \%)$ des effectifs de ce groupe professionnel. Le violon, à lui seul, en rassemble un tiers $(32,9 \%)$. Cette prédominance des cordes se retrouve par ailleurs dans les conditions d'accès à l'emploi.

En dépit de l'absence de restriction lors de l'inscription à la majorité des concours d'orchestre (Ravet, 2006) empêchant d'évaluer le nombre exact des postulants ${ }^{8}$, les diplômés du Conservatoire national supérieur de musique (CNSM) de Paris per-

6. Source: Programme de la saison 2011/2012 de l'Orchestre de Paris disponible sur son site Internet www.orchestredeparis.com/

7. Ce recensement a été effectué en 2011 à partir des listes de musiciens disponibles sur les sites Internet des 29 orchestres permanents membres de l'AFO.

8. On retrouve la même absence de restriction initiale dans les procédés de sélection en danse contemporaine (Sorignet, 2004a). 
mettent toutefois d'effectuer des comparaisons instrumentales sur les chances d'intégrer un orchestre. Ces derniers peuvent servir d'échelle de référence fiable, dans la mesure où ils sont les produits (avec les diplômés du CNSM de Lyon) du système pyramidal français de formation musicale ${ }^{9}$ destinés aux emplois de permanents d'orchestre. La comparaison par instrument entre les emplois potentiels dans les orchestres et le nombre des diplômés du CNSM de Paris de 2003 à 2006 ${ }^{10}$ (voir Tableau 1 et Graphique 1) est alors un outil pour appréhender la question de l'inégalité instrumentale d'accès aux emplois de permanent. Plus le rapport effectifs de permanents/diplômés du CNSM est élevé, plus les diplômés ont des potentialités d'emploi importantes et donc plus les chances d'accès à l'orchestre sont conséquentes.

\section{Tableau 1: Répartition par instrument des permanents d'orchestre et des diplômés du CNSM de Paris}

\begin{tabular}{|l|c|c|}
\hline Instrument & Effectifs de permanents d'orchestre & Diplômés du CNSM de Paris 2003/2006 \\
\hline violon & 658 & 97 \\
\hline alto & 243 & 43 \\
\hline violoncelle & 208 & 61 \\
\hline contrebasse & 147 & 13 \\
\hline flûte traversière & 86 & 22 \\
\hline hautbois & 87 & 17 \\
\hline clarinette & 89 & 28 \\
\hline basson & 84 & 19 \\
\hline cor & 121 & 14 \\
\hline trompette & 85 & 17 \\
\hline trombone & 71 & 15 \\
\hline tuba & 14 & 13 \\
\hline percussions & 81 & 20 \\
\hline harpe & 19 & 9 \\
\hline piano & 10 & 116 \\
\hline Total & 2003 & 504 \\
\hline
\end{tabular}

Sources: Pour les effectifs de permanents: recensement effectué par nos soins à partir des listes de musiciens disponibles sur les sites Internet des 29 orchestres permanents affiliés à l'AFO; pour les diplômés du CNSM de Paris: Palmarès 2003, 2004, 2005, 2006 édités par le CNSM de Paris.

9. Le système pyramidal de l'enseignement musical français a été adopté en 1969 et renforcé depuis par toutes les législations. Cette organisation vise, par l'octroi de différents agréments délivrés par le ministère de la Culture, à classifier, ordonner et hiérarchiser les différentes institutions de formation musicale. Au sommet, figurent les CNSM de Paris et de Lyon chargés de former les professionnels de la musique classique. Puis viennent, par ordre décroissant, les 36 conservatoires à rayonnement régional (CRR), les 101 conservatoires à rayonnement départemental (CRD), les 314 conservatoires à rayonnement communal (CRC) et les quelque 3000 écoles de musique non agréées, qui sont chargés, à des degrés divers, de former des amateurs éclairés (Cueille, 2004; Ganvert, 1999).

10. La prise en compte des effectifs cumulés de diplômés pendant quatre années évite toute forme de biais dans le calcul induit par une «sur» ou une «sous» représentation selon les années. 


\section{Graphique 1: Rapport par instrument entre postes potentiels de permanents d'orchestre et diplômés du CNSM de Paris}

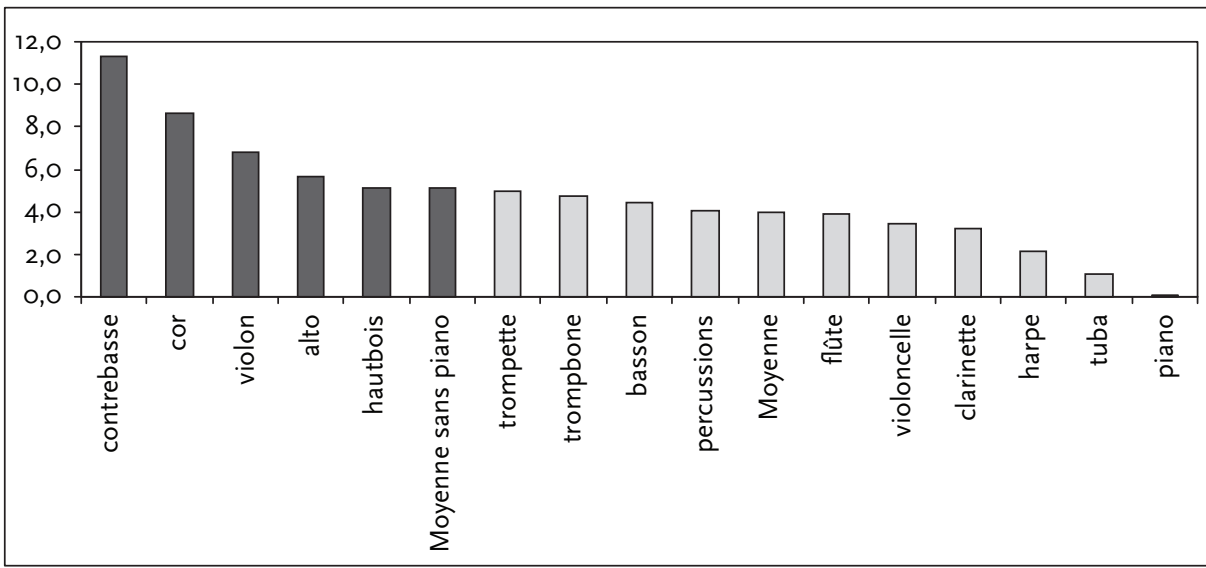

La faiblesse des postes en piano conjuguée nombre important de diplômés du CNSM dans cet instrument pouvant induire une moyenne tronquée, nous avons fait apparaître sur le graphique la moyenne sans le piano plus efficiente pour rendre compte des rapports moyens.

Sources: Idem que Tableau 1.

Cette comparaison souligne les divergences instrumentales des possibilités d'accession aux emplois de permanents d'orchestre. Pour les interprètes du piano, du tuba et de la harpe, les opportunités potentielles d'emploi dans les orchestres sont extrêmement faibles. À l'opposé, les musiciens pratiquant la contrebasse, le cor, le violon et l'alto sont privilégiés. D’un point de vue général, les cordes sont ainsi en situation favorable puisqu'elles constituent tout de même trois des quatre instruments qui offrent des possibilités accrues d'accession aux postes de permanents d'orchestre.

\section{Inégalités instrumentales et hiérarchies sociales entre les interprètes}

Même si des disparités s'observent entre les instruments, les interprètes de la famille des cordes se retrouvent tendanciellement favorisés pour l'obtention des postes de musiciens indépendants et de permanents d'orchestre par rapport à leurs homologues des autres familles instrumentales. Ces inégalités instrumentales d'accès aux positions dominantes du champ de la musique classique reproduisent alors les inégalités sociales de leurs interprètes.

En effet, plusieurs études menées sur les instrumentistes classiques professionnels français ont souligné la différenciation sociale entre les familles instrumentales. Les données recueillies par Philippe Coulangeon (voir 2004 : 115) révèlent ainsi que 50 \% des interprètes professionnels des instruments à cordes sont originaires des classes supérieures, cette proportion étant de $44 \%$ pour les bois et $31 \%$ pour les cuivres. La part des enfants des classes populaires y est d'environ un tiers pour les cordes, de $31 \%$ pour les bois et de $44 \%$ pour les cuivres. Les travaux de Bernard Lehmann (voir 2005: 49) sur les permanents d'orchestre soulignent une inclination similaire: 46,2\% des 
musiciens jouant d'instruments à cordes sont issus des classes supérieures, contre $40 \%$ pour les bois et $20 \%$ pour les cuivres, la part des enfants des classes populaires étant respectivement pour ces trois familles instrumentales de $11,5 \%, 28 \%$ et $40 \%$. Les résultats de notre enquête consacrée aux instrumentistes classiques d'une ville de province (Pégourdie, 2013) ${ }^{11}$ s'inscrivent dans une tendance analogue. Les proportions des musiciens provenant des classes supérieures et des classes populaires sont de $57,1 \%$ et $16 \%$ pour les instrumentistes à cordes, de $37,5 \%$ et $37,5 \%$ pour les instrumentistes des bois et de $28,6 \%$ et $42,9 \%$ pour les instrumentistes des cuivres.

Cette différenciation sociale de la pratique instrumentale professionnelle, qui se double comme l'ont montré plusieurs recherches d'une différenciation sexuelle (Escal et Rousseau-Dujardin, 1999; Ravet, 2003; Coulangeon et Ravet, 2003; Monnot, 2012), contribue à distinguer des cordes, qui représentent un groupe féminisé et socialement sélectif, des bois, qui constituent une position médiane tant par l'importance des musiciens originaires des classes moyennes que par la répartition équivalente entre les sexes, et des cuivres très masculins et à connotation sociale plus populaire.

Si ces groupes ne doivent pas être considérés comme figés, car ils tolèrent des exceptions et ne fonctionnent que par différences relationnelles, par variations de la proportion des enfants des classes supérieures et/ou de la représentation féminine, les différenciations sociales qu'ils révèlent n'en demeurent pas moins observables dans toutes les enquêtes sur les institutions musicales. Cette permanence s'ancre dans l'histoire du champ français de la musique classique. En effet, dès la Révolution française, jouer des instruments à cordes et à vent (dénomination qui regroupe les bois et les cuivres) tend à être socialement différencié. Les instruments à cordes, envisagés dès le $\mathrm{XIX}^{\mathrm{e}}$ siècle comme les modèles de l'approche artistique en musique classique, participent de ce fait à l'autonomisation du champ ${ }^{12}$ et s'opposent nettement aux instruments à vent, initialement appréhendés comme relevant de la musique militaire (Leterrier, 1999; Ganvert, 1999; Lehmann, 2005). Cette différenciation primaire est renforcée par les diverses formes de socialisation musicale au cours du XIx ${ }^{e}$ siècle. L'apprentissage des instruments à cordes, en particulier le violon et le piano, profondément intégré dans la culture bourgeoise (Menger, 2001), et notamment dans l'éducation traditionnelle des jeunes filles de ce milieu (Lenoir, 1979), se distingue alors de l'enseignement des instruments à vent lié au mouvement orphéonique et tourné vers les classes populaires et la population masculine (Gerbod, 1980; Gumplowicz, 2001; Dubois et al., 2009). Les cordes, en tant qu'instruments de distinction de la culture des couches dominantes, s'affilient à une vision conjointement bourgeoise et féminine, tandis que les vents se parent des attributs d'une culture inséparablement populaire et masculine.

Ces principes de division sociale et sexuelle des instruments ne s'appuient néanmoins pas uniquement sur des processus de différenciation liés à l'histoire du champ.

11. Pour la méthodologie de l'enquête, voir l'encadré en amont.

12. Sur les processus d'autonomisation d'un champ artistique par la figure de l' "art pur», voir Bourdieu $(1975,2013)$. 
S'ils conservent leur pertinence et leur acuité, c'est également parce que les différenciations initiales sont réactivées dans la pratique à travers toute une série d'oppositions ternaires séparant les cordes, les bois et les cuivres qui contribuent à une division du monde instrumental en classes logiques. Bernard Lehmann (2005) a ainsi démontré comment la mise en scène du corps dans l'interprétation (les cordes se jouent avec la médiation d'un archet tandis que les bois et les cuivres sont des instruments que l'on embouche, par l'intermédiaire d'une anche pour les bois, directement pour les cuivres), la matière dont sont faits les instruments (à la «noblesse» du bois dont sont faits les instruments à cordes s'oppose la «trivialité» du métal des cuivres, les bois étant à nouveau en position intermédiaire), la tessiture des notes proposées (à l'aigu des cordes correspond la mélodie, au grave des cuivres l'accompagnement ${ }^{13}$ et la disposition spatiale des instruments dans l'orchestre (les cordes à l'avant, les bois au centre, les cuivres à l'arrière) incitent à une séparation franche entre des univers musicaux sexuellement et socialement connotés faisant sens pour les interprètes.

On constate ainsi une forme d'homologie entre les conditions d'accès aux postes les plus légitimes du champ de la musique classique et la division sociale du travail instrumental ${ }^{14}$. Plus un instrument se caractérise par un recrutement social élevé, plus les possibilités d'obtention d'une position dominante y sont importantes. Les formes routinisées de la consécration musicale ne relèvent donc pas uniquement d'une logique «artistiquement pure» célébrant le «talent» ou les compétences propres aux instrumentistes mais empruntent la voie de la consécration sociale en distinguant principalement les interprètes des instruments les plus sélectifs socialement.

La restriction des conditions d'accès à une position légitime pour les interprètes des instruments à la connotation la moins bourgeoise ${ }^{15}$, signe d'une reproduction sociale au sommet du champ de la musique classique, induit une surqualification de ces interprètes sur les positions intermédiaires. Soumis à des sélections draconiennes, voire exclus des postes les plus légitimes, des instrumentistes détenteurs d'un important capital propre au champ de la musique classique (diplôme des CNSM, réussite à des concours internationaux notamment) ne peuvent pourtant prétendre aux formes routinisées de la consécration musicale. Ils s'insèrent alors dans des postes moyens ${ }^{16}$,

13. Bernard Lehmann (2005) souligne également que la tessiture conduit à distinguer les instruments au sein de leur propre famille instrumentale: le continuum aigu/grave, et donc mélodie/accompagnement, distingue ainsi les cordes du violon à la contrebasse, les bois de la flûte traversière au basson, etc.

14. Cette homologie se retrouve sur l'ensemble des positions du champ de la musique classique, et notamment sur les postes d'enseignant, proportionnellement plus nombreux et aux meilleures conditions de travail (emploi à temps complet dans un seul conservatoire par exemple) pour les instruments à cordes (Pégourdie, 2013).

15. Cette situation touche également certains interprètes d'instrument au recrutement socialement élevé, tels les harpistes dont nous traiterons un cas plus loin. La particularité de la harpe peut s'expliquer par la féminisation importante de cet instrument, facteur discriminant dans un secteur professionnel marqué par une ségrégation sexuelle verticale (Ravet, 2003; Coulangeon et Ravet, 2003).

16. On assiste alors sur ces positions intermédiaires à un renversement des hiérarchies. Les instruments dominant socialement y sont, dès lors, dominés musicalement. Ce mécanisme est analogue à celui décrit par Bernard Lehmann (2005) pour les orchestres symphoniques. À l'orchestre, peu d'instrumentistes 
notamment des emplois d'enseignants ${ }^{17}$ dans des conservatoires situés dans les échelons supérieurs de la pyramide de l'enseignement musical (cf. supra) ${ }^{18}$. Après un apprentissage particulièrement long et ascétique couronné par les classements les plus positifs, ceux qui certifient l'excellence musicale et nourrissent logiquement autant la croyance individuelle dans le «talent» que l'espoir de l'accès aux positions dominantes du champ musical, il reste alors pour eux à se faire à cette situation intermédiaire. Pour cela, ils vont trouver dans la réinterprétation des positions intermédiaires du champ, et en particulier de l'activité pédagogique, un moyen de surmonter personnellement le deuil de leurs aspirations déchues à la consécration et d'aménager une forme de gestion de leur insuccès dû aux dynamiques sociales de la reconnaissance musicale.

\section{SURMONTER L'ABSENCE DE RECONNAISSANCE}

La gestion de l'insuccès ne s'exprime néanmoins pas de la même façon pour tous les instrumentistes. Parvenir à faire le deuil de la reconnaissance musicale et éviter le ressentiment lié à l'accès à une position moyenne, processus d'autant plus complexes dans des métiers artistiques qui par leur régime vocationnel entretiennent le mythe de l'élection (Sapiro, 2007), passent par une réévaluation de l'activité et du statut d'enseignant. Cette réévaluation s'opère différemment selon les propriétés sociales des agents et leur inscription dans le champ. En ce sens l'origine sociale, le sexe et la proximité précoce avec le champ de la musique classique des instrumentistes, en orientant les trajectoires et la manière de «se faire à sa position ", participent à la différenciation des modalités de réinterprétation de l'activité pédagogique. Ainsi, ces propriétés sociales déterminent autant les conditions d'accès à la reconnaissance musicale que la façon de surmonter le fait d'en être privé.

à cordes peuvent accéder au statut de soliste et doivent donc se rabattre sur la fonction de tuttiste. À l'opposé, tous les vents ont un statut de soliste. Cette situation provoque un sentiment de déclassement chez les interprètes des cordes non héritiers qui les conduit parfois au désenchantement (Adenot, 2008).

17. L'accès au statut d'intermittent du spectacle, autre position intermédiaire du champ de la musique classique, est encadré par des logiques spécifiques que nous avons traitées dans un autre article (Pégourdie, 2012).

18. Il s'agit ici des 36 conservatoires à rayonnement régional (CRR) qui constituent le second échelon de la pyramide de l'enseignement musical français. Les deux CNSM de Paris et Lyon, qui représentent le premier échelon de cette pyramide, ont des logiques de recrutement des enseignants particulières qui en font des positions dominantes dans le champ. Centrés sur la production de musiciens professionnels, les CNSM recrutent des musiciens indépendants ou des permanents d'orchestre, donc des instrumentistes qui ont déjà accédé aux formes routinisées de la reconnaissance. Confrontés à des élèves au niveau instrumental relativement élevé, les pédagogues doivent être en mesure, pour asseoir la légitimité de leur enseignement, de démontrer leur supériorité instrumentale et d'en «imposer» par leur parcours professionnel. Ce recrutement particulier participe également à l'affirmation de la place d'élite de ces conservatoires. En recrutant des spécialistes unanimement salués, ces établissements confirment leur affiliation à la filière d'excellence. En ce sens, il est logique que ces mêmes conservatoires favorisent et encouragent le maintien des prestations artistiques. L'activité artistique des musiciens est ainsi au principe de leur recrutement dans les conservatoires d'élite et, par là, vient renforcer la consécration de l'instrumentiste. Ces éléments expliquent pourquoi l'enseignement dans ces conservatoires d'excellence n'a besoin d'aucune forme de réinterprétation. 
L'étude des trajectoires de deux enseignants d'un conservatoire à rayonnement régional (CRR), établissement affilié au deuxième des cinq échelons de la pyramide de l'enseignement musical français (cf. supra) ${ }^{19}$, permet de saisir les effets des propriétés sociales et de la pratique d'un instrument particulier dans le processus d'ajustement aux positions moyennes. Il s'agit d'Yves, un saxophoniste, et de Christine, une harpiste, qui représentent deux «configurations singulières» (Lahire, 1995) ${ }^{20}$, celle d'un enfant des classes supérieures non sensibilisées à la musique qui s'oriente vers un instrument socialement peu sélectif et celle d'une «héritière» qui ne parvient pas à reproduire complètement la position de ses parents. Détenteurs d'un important capital spécifique (diplômés des CNSM et lauréats de concours internationaux), ils occupent tous les deux une position intermédiaire d'enseignant, du fait de leur pratique d'instruments aux faibles possibilités d'accès aux positions dominantes du champ — l'un joue d'un instrument disqualifié sur le marché de la musique savante, l'autre d'un instrument très valorisé mais sans marché. Représentant au sein de l'échantillon enquêté des instrumentistes aux aspirations déchues qui doivent surmonter l'absence d'une consécration que leur parcours de formation semblait leur offrir ${ }^{21}$, leurs trajectoires se distinguent car ils entretiennent, par l'effet de leurs différences sociales initiales, deux rapports opposés à l'activité pédagogique, de l'enseignement «sous contrainte» à la valorisation artistique par la pédagogie. En ce sens, ils proposent deux modes de revalorisation d'une position moyenne où la recherche de confirmation de leur «talent» s'éloigne des formes routinisées de consécration tout en prenant des acceptions différenciées.

\section{Nécessité d'enseigner et salut hors du conservatoire}

Yves, cadet d'une famille de cinq enfants, est né dans la région lyonnaise en 1973. Son père, informaticien, et sa mère, professeure de mathématiques puis femme au foyer à la naissance de son troisième enfant, sont mélomanes. C'est donc, selon lui, tout naturellement qu'il commence l'apprentissage de la musique, imitant ainsi trois de ses aînés. À sept ans, il intègre un conservatoire à rayonnement de la région lyonnaise, puis se met à apprendre le saxophone après un an de solfège. Il présente le choix de cet instrument comme une décision personnelle liée à un «coup de cour» survenu à six ans, alors qu'il assiste à un concert pendant un voyage en Autriche. Yves envisage très précocement une carrière musicale: «Alors en fait, moi, depuis l'âge de huit ans, j'ai décidé de faire de la musique.» Ses parents l’inscrivent dans une classe à horaires amé-

19. Ces deux trajectoires sont extraites du travail de thèse dont la méthodologie a été précisée an amont (Pégourdie, 2013).

20. Ces cas, compte tenu de la structure de l'échantillon, ne sauraient être envisagés comme des idéaux-types. Le modèle des «configurations singulières», qui s'attache à rendre compte de la combinaison des facteurs plutôt que de l'effet de chaque facteur pris isolément, est ainsi paru plus adapté pour cette tâche.

21. Tous les interprètes enquêtés ne présentent pas les mêmes caractéristiques. Pour la majorité des enseignants du conservatoire étudié, l'accès à cette position moyenne est conforme à leur volume moyen de capital spécifique. Ajustés à leur position, ils ne sont pas soumis à la même tension concernant leur absence de reconnaissance. 
nagés musique (CHAM) qui lui permet de mener de front son cursus en saxophone classique au conservatoire de Lyon et sa scolarité.

À 14 ans, il a déjà achevé les trois cycles de formation en saxophone au conservatoire. Il poursuit néanmoins les cours avec son professeur lyonnais qui crée, pour lui et quelques-uns de ses camarades, une formation pré-professionnelle en saxophone. Il se perfectionne ainsi pendant quatre ans jusqu'à l'obtention de son baccalauréat F11, c'est-à-dire option musique. Dans le même temps, il entreprend à 17 ans des récitals solistes et donne quelques cours particuliers. Il apprend également la contrebasse, suivant les conseils de son professeur, qui lui recommande de s'initier à un instrument d'orchestre pour se ménager des possibilités complémentaires d'emploi ${ }^{22}$. À l'âge de 20 ans, il réussit le concours d'entrée du CNSM de Paris. Il achève ses études trois ans plus tard en obtenant le premier prix à l'unanimité ${ }^{23}$ et le certificat d'aptitudes aux fonctions de professeur de musique (CA $)^{24}$. Pendant cette période, il fonde, avec trois de ses camarades du CNSM, un quatuor de saxophones spécialisé dans le répertoire strictement classique, c'est-à-dire sans jazz ni «musiques actuelles $»^{25}$.

Ce jeune quatuor parvient à se faire une «petite réputation» dans le milieu musical, en se produisant à plusieurs reprises, sans toutefois pouvoir réunir assez de cachets pour prétendre au statut intermittent ${ }^{26}$. Yves refuse alors de se tourner vers la variété ou le jazz qui auraient pu lui accorder des cachets complémentaires lui permettant l'accession à l'intermittence. Malgré son CA, il n'entend pas non plus orienter son activité professionnelle vers l'enseignement dès sa sortie du CNSM.

Le refus initial d'une carrière pédagogique et l'exclusivité revendiquée d'une pratique instrumentale dans un genre esthétique mineur en saxophone traduisent alors le rapport au métier entretenu par Yves à la fin de ses études, rapport façonné par ses propriétés sociales et son cursus musical.

Élève précoce et brillant, considérant jouer du saxophone sur le mode vocationnel, il a durant toute sa formation été animé de la conviction, basée sur le principe mystique de l'élection, de son «don» pour le saxophone ${ }^{27}$. Cette conviction, entretenue par son

22. Les potentialités importantes d'accès aux postes d'orchestre en contrebasse soulignent l'efficience de ce conseil (cf. supra Graphique 1).

23. Le Premier Prix à l'unanimité est la plus haute distinction décernée par le CNSM. Suivent par ordre décroissant le premier prix, le deuxième prix à l'unanimité, le deuxième prix, le troisième prix à l'unanimité et le troisième prix. Par comparaison, l'année suivante, en 1994, sur les six diplômés de saxophone du CNSM, seule la moitié obtient un premier prix à l'unanimité. Source: Conservatoire national supérieur de musique et de danse de Paris, Palmarès 1994, p. 72.

24. Le CA est le diplôme d'enseignement musical français le plus élevé. Il devance le Diplôme d'État de professeur de musique (DE). La différence entre ces deux diplômes est similaire à celle que l'on peut trouver dans l'enseignement secondaire entre l'agrégation et le CAPES.

25. La catégorie «musiques actuelles» est une catégorie institutionnelle créée dans les années 1980 par le ministère de la Culture pour traiter tout un ensemble de genres musicaux qui se définit avant tout par son opposition à celui des «musiques savantes» : rock, musiques électroniques, musiques du monde, jazz, rap, variétés, etc. (Teillet, 2003).

26. Sur les difficultés d'accès au statut intermittent à partir des années 1990, voir Menger (2002b).

27. Pour une présentation générale des travaux sociologiques traitant des rapports entre discours vocationnel et idéologie du don, se référer à Gisèle Sapiro (2007). Pour une présentation de la notion de vocation dans l'univers culturel, voir Vincent Dubois (2013). 
professeur lyonnais ${ }^{28}$, notamment à travers l'enseignement reçu dans la classe pré-professionnelle, et par ses études au CNSM, a été à l'origine de l'incorporation d'aspirations musicales élevées, associant la réussite professionnelle à un accomplissement artistique seulement atteignable par des prestations musicales classiques. Issu d'un milieu favorisé mais éloigné de l'univers professionnel de la musique, Yves n’a en outre pu trouver dans son environnement familial de régulateur pour ses aspirations musicales, non ajustées aux possibilités concrètes d'emploi pour un saxophoniste. Devant faire face, à la fin de ses études, à la réalité du marché du travail en tant que saxophoniste, Yves fait alors l'amer constat de la démesure de ses ambitions. Ses prestations de saxophone classique, malgré la qualité reconnue de son quatuor, ne suffiront pas, à elles seules, à lui procurer un revenu suffisant. Pour vivre de la musique, il doit aller chercher des cachets dans d'autres genres musicaux ou enseigner. Avec cet instrument, la radicalité stylistique n'est «rentable» que symboliquement, aucun sous-espace de la musique savante ne venant lui accorder de débouchés professionnels.

Son insertion professionnelle marque donc la recomposition de sa croyance en ses aspirations musicales élevées, associées à une image mythifiée de l' "artiste pur» (Bourdieu, 1975) ${ }^{29}$. Cette prise de conscience s'accompagne d'un discours critique envers le système de formation et les croyances improbables qu'il véhicule: «Donc on se demande où vont tous ces gens qui partent à Paris. Qu'est-ce qu'ils font après? Si je regarde au niveau des saxophonistes, tous les trois ans, y'a douze personnes qui sortent, donc on va dire une moyenne de quatre par an sur trois ans. Les quatre personnes qui sortent tous les ans en moyenne d'un conservatoire de Paris en saxophone, ils font quoi ces gens? Et sur ceux que je connais, on va dire sur les dix dernières années, ça représente quarante personnes. Qui sont actifs aujourd'hui? S’il y en a la moitié, c'est bien le maximum, enfin qui sont actifs et qui proposent des choses au niveau saxophone. Alors les autres, on sait pas ce qu'ils font. Ils donnent des cours quelque part dont on n'entend plus jamais parler parce qu'ils ont pas forcément envie, je sais pas. (...) Et les places, elles se font rares parce qu'il y a une cinquantaine de conservatoires en France et les gens en place aujourd'hui beaucoup ont moins de 40 ans. Ce qui veut dire qu'au moins pendant 20 ans, y'a plus de place. Et ces gens vont faire quoi? Le niveau monte au CNSM à Paris, comme toujours, ça a toujours été comme ça, un niveau formidable. Plus ça va, mieux c'est. Sauf qu'on n'a rien à leur promettre à ces gens-là. Alors ils vont se rabattre sur quoi? Sur les écoles de musique ou les écoles agréées de moindre importance que les CRR parce qu'ils auront pas le choix. (...) On va dire sur les quarante personnes qui sortent sur les prochaines années, y'en a deux ou trois qui arrivent à percer et à être solistes. À mon avis, ça n'existe pas encore. Il y en a quelques-uns mais ils ont pas une carrière de soliste, ils ont une carrière de prof plus de soliste.»

28. Sur l'action des institutions de formation dans l'incorporation d'une vocation, voir Charles Suaud (1978).

29. Cette question de la recomposition, et des modalités sociales de cette recomposition, de la référence au mythe de l' «artiste pur» au fur et à mesure de l'avancée dans la carrière a également été traitée par Pierre-Emmanuel Sorignet dans le cas des fins de carrière des danseurs contemporains (Sorignet, 2004b). 
Pour Yves, cette désillusion s'avère difficilement surmontable. La force de l'incorporation de ses aspirations musicales l'empêche de percevoir la diversification stylistique ou la pédagogie autrement que comme des compromissions artistiques. Plutôt que d'abandonner ses ambitions en s'engageant dans une carrière intermittente ou enseignante, il décide de quitter le quatuor et la France pour «tenter l'aventure» de la vie anglaise avec sa compagne de l'époque. Ne pouvant se soustraire à un renoncement à son idéal artistique, il entend «conserver son intégrité» en appliquant, à sa vie personnelle, le mode de vie artiste, qu'il perçoit comme légitime, auquel il ne peut prétendre dans sa vie professionnelle ${ }^{30}$. Son départ lui permet de se représenter symboliquement comme un «artiste pur», ne pouvant se résoudre à la moindre concession et privilégiant le retrait du monde musical français et le désintéressement.

Ces dimensions s'expriment d'ailleurs dès son arrivée en Angleterre. Il éprouve des difficultés à vivre de la musique et doit se résoudre pendant un an et demi à exercer le métier de serveur dans un pub. Il continue toutefois à jouer intensivement de son instrument. Sa persévérance est alors récompensée. Il décroche deux deuxièmes prix lors de concours internationaux renommés à Bayreuth et à Birmingham, puis remporte un concours en Angleterre lui permettant de se produire en soliste dans une dizaine de concerts avec un orchestre amateur. Il donne également des cours et participe à l'organisation d'un concours. C'est à ce moment que ses anciens partenaires le contactent pour reformer le quatuor. Il répond favorablement à leur demande. Pendant une année, il multiplie les aller-retour entre Paris et l'Angleterre. Toutefois, l'accroissement de l'activité du quatuor et la séparation avec sa compagne l'incitent finalement à rentrer en France après six années passées outre-Manche. Convaincu de l'impossibilité de dégager des revenus suffisants de sa participation au quatuor, il décide à son retour d'embrasser la carrière pédagogique.

Son expérience anglaise peut être considérée comme une étape nécessaire lui ayant permis d'accepter symboliquement la nécessité d'enseigner, sans avoir l'impression de se renier. Titulaire du CA depuis la fin de ses études au CNSM, il postule à quatre postes de professeur de saxophone. Il est recruté pour l'un d'eux et devient, en 2002, professeur d'enseignement artistique titulaire, c'est-à-dire fonctionnaire territorial, dans un CRR de province. Il maintient en parallèle son activité au sein du quatuor avec lequel il répète une fois par semaine à Paris. L'investissement des quatre saxophonistes dans cet ensemble est alors salué. Ils sont lauréats d'un deuxième prix au Concours international de musique de chambre d'Osaka, obtiennent plusieurs subventions de la part d'organismes privés et publics et se produisent régulièrement en France et quelques fois à l'étranger.

S’il obtient enfin une considération artistique avec son ensemble, elle ne s'accompagne toutefois pas d'un abandon de son emploi enseignant. Yves a progressivement intériorisé la nécessité d'enseigner pour se dégager, en tant que saxophoniste, un

30. Philippe Coulangeon soulève une pareille confusion dans la perception artistique de la vie personnelle et de la vie professionnelle chez les musiciens de jazz qui tendent à faire de «leur existence quotidienne une œuvre de génie» (Coulangeon, 1999: 693). 
revenu constant dans l'univers de la musique classique ${ }^{31}$. Marié depuis quelques années à une enseignante de saxophone et père d'une petite fille depuis 2007, il considère la pédagogie, non comme une pratique source d'épanouissement artistique, mais bien comme un moyen d'obtenir un salaire mensuel stable ${ }^{32}$ lui permettant, sans se renier, de se consacrer ponctuellement à ses activités musicales «enrichissantes » : le quatuor, les prestations solistes et quelques engagements dans un orchestre en qualité de contrebassiste supplémentaire. Activité essentiellement alimentaire, l'enseignement s'apparente à une «diversification périphérique» (Coulangeon, 1999: 706) qui ne cadre pas avec sa vision de l'artiste musicien. Son salut se rencontre dans ses prestations en dehors du conservatoire: "J'aurais voulu gagner ma vie qu'avec la musique. Ça n’a pas été possible parce que mon cas est un peu particulier. Je suis saxophoniste et le saxophone classique n'a que 160 ans et est donc inconnu du public. Et donc comment voulez-vous que je remplisse une salle en jouant du Glazounov ou du Schmitt? (...) Donc j'aurais voulu être musicien. Mais de toute façon je savais, on m'a vite dit, et c'est très bien, que si je voulais faire du saxophone et faire des concerts, il faudrait que je gagne ma vie de l'enseignement. C'est pas possible autrement. (...) Alors pourquoi j'ai choisi de faire ça plutôt qu'autre chose, j'en sais rien. C'est comme ça, on choisit de faire quelque chose et puis après on se fait prendre dans les méandres du fonctionnement, et on n'arrive pas forcément là où on voulait mais on en gagne sa vie quand même.»

\section{Se valoriser artistiquement par l'enseignement}

Christine, née en 1950, est issue d'une famille de musiciens parisiens. Fille et sœur de musiciens professionnels (son père était un chef d'orchestre renommé et son frère violoniste), elle est également femme et mère d'instrumentistes. Son mari est altiste et ses deux filles sont musiciennes: l'une en violon dans un orchestre autrichien, la seconde achève sa formation professionnelle en violoncelle. Seul son fils, qui a néanmoins un bon niveau en harpe, n'est pas engagé dans une carrière musicale.

31. Ces ajustements progressifs, permettant de se faire à une position ou à une façon d'occuper une position différente de celles qui avaient été envisagées lors de la période de formation, se rencontrent également chez les professeurs du secondaire (Balland, 2012).

32. Cette stabilité des revenus des enseignants de musique n'est pas partagée par tous les pédagogues du secteur artistique. Sébastien Fleuriel, qui traite lui aussi des tensions entre pratique artistique et activité pédagogique et du caractère subsidiaire des revenus de l'enseignement dans l'esprit des danseurs de jazz, souligne à l'inverse la précarité des enseignants de ce secteur artistique (Fleuriel, 2004). Ces différentes conditions s'expliquent par les modalités d'accès à la titularisation. Ainsi la stabilité professionnelle des enseignants de musique est liée à leur intégration massive à la fonction publique territoriale, décidée en 1991, et qu'il faut appréhender comme le résultat de politiques étatiques menées, depuis les années 1970, par la Direction de la musique et de la danse (DMD), organe du ministère de la Culture, visant conjointement au développement de l'offre d'enseignement musical et à la standardisation et la normalisation des conditions de recrutement, d'emploi et de rémunération des enseignants de musique dans le but d'une amélioration de la «qualité» de l'enseignement. Sur ce point, voir les travaux menés dans la thèse de Pégourdie (2013). Pour une vision d'ensemble du rapport de l'État à la culture, se référer à l'ouvrage de Vincent Dubois (1999). 
Cet environnement explique ses débuts musicaux précoces. Ainsi, dès six ans, elle apprend le violon dans un conservatoire parisien. Elle l'abandonne rapidement pour se consacrer à l'étude du piano. Pendant une dizaine d'années, elle se perfectionne dans la technique de cet instrument, allant même jusqu'à être inscrite au concours d'entrée du CNSM de Paris. Mais l'année de ses 16 ans, alors qu'elle prépare activement son examen d'entrée en piano, elle fait la rencontre d'une enseignante de harpe. La découverte de cette pédagogue et de cet instrument agit chez elle comme une «révélation». Elle abandonne le piano et se lance à corps perdu dans l'apprentissage de la harpe ${ }^{33}$. Deux ans lui suffisent pour réussir le concours et intégrer le CNSM en classe de harpe. L'image mythifiée de ses débuts en harpe, puis le sentiment d'élection qui découle de ses progrès fulgurants dans cet instrument sont largement repris dans ses propos: «Ah! le violon, je l'ai fait très peu d'années au départ. Quand j'étais très petite, vers six-sept ans. Après, j'ai fait une dizaine d'années de piano. Et puis j'ai commencé très tard la harpe. Ça a été un peu un challenge, un défi parce que c'était l'année où je me présentais au CNSM à Paris en piano. Et puis finalement, l'année où je devais me présenter, j'ai rencontré une harpiste. Et là, j'ai changé totalement de projet. Donc en deux ans, j'ai appris la harpe. Et je me suis présentée au bout de deux ans et demi au CNSM et je suis rentrée à la harpe. (...) Quand on a la chance d'avoir un père chef d'orchestre et qu'on voit les concerts à l'orchestre, eh bien on veut faire partie de ça. Et je crois que ça m'a un peu orientée pour essayer de changer d'instrument. Mais ça a été plutôt la rencontre d'un professeur qui m'a fait comprendre que j'étais plus faite pour faire de la harpe que du piano. Donc ça a été un peu le coup de foudre.»

À 18 ans, son baccalauréat obtenu, elle intègre la classe de harpe du CNSM. Ses études se déroulent alors de manière relativement linéaire. Elle obtient le premier prix à l'unanimité en harpe, puis, deux ans plus tard, le premier prix en musique de chambre. Elle connaît également ses premières expériences professionnelles grâce aux relations tissées au CNSM et aux réseaux de ses parents. En 1976, à 26 ans, elle quitte l'institution parisienne et entre sur le marché du travail.

Malgré ses quelques expériences professionnelles étudiantes, elle éprouve alors de grandes difficultés d'insertion. Elle subit de plein fouet une âpre concurrence aussi bien dans le domaine de l'interprétation que dans l'enseignement. Compte tenu de la faiblesse des emplois offerts (cf. supra Graphique 1), la perspective d'intégrer un orchestre symphonique lui apparaît immédiatement comme un avenir professionnel peu probable. La difficulté est similaire en ce qui concerne les formations de musique de chambre et les prestations solistes. Si des engagements sont ponctuellement possibles — Christine en effectuera d'ailleurs durant toute sa carrière —, leur volume est loin d'être suffisant pour accéder au régime de l'intermittence. C’est donc vers l'enseigne-

33. Si Christine change trois fois d'instrument, passant du violon au piano puis à la harpe, elle demeure toutefois toujours dans les «cadres» de la division sexuelle des instruments (Coulangeon et Ravet, 2003). Sur les modes d'orientation des jeunes filles vers les instruments féminins, voir Catherine Monnot (2012). 
ment que Christine, consciente dès ses études de la difficulté à gagner sa vie avec la seule interprétation, décide d'orienter son activité professionnelle.

Cependant, le secteur pédagogique lui réserve également de grandes difficultés d'insertion professionnelle. L'enseignement de la harpe est très peu développé lorsqu'elle quitte le CNSM en 1976. Il n'existe aucun diplôme certifiant la compétence pédagogique du musicien. Le CA de harpe n'a pas encore été créé, alors que cette certification est délivrée depuis 1969 pour d'autres instruments. De plus, seuls quelques rares conservatoires offrent des cours, l'essentiel de l'offre pédagogique étant assuré par des professeurs particuliers, destinant leurs savoirs à des futurs professionnels. La faiblesse de l'offre de formation en harpe, si elle est aujourd'hui moins criante que lors des débuts professionnels de Christine, demeure néanmoins encore réelle. La harpe n'est enseignée que dans 95 conservatoires sur les 136 CRR et CRD, ce qui la place au troisième rang des instruments les moins diffusés, derrière l'accordéon et la flûte à bec. En outre, l'effectif des enseignants de harpe ne s'y élève qu'à 121, soit le nombre le plus faible avant l'accordéon (84) et le tuba (116). À titre de comparaison, les enseignants de piano, les plus nombreux, sont 949. Pour juger du différentiel avec la trajectoire précédente, les enseignants de saxophone sont au nombre de $250^{34}$.

L'obtention d'un poste d'enseignant dans les conservatoires offrant des cours de harpe s'avère alors complexe, les lois féroces de la concurrence s'appliquant de manière quasi équivalente aux emplois de permanent d'orchestre. Christine s'applique donc à démarcher les conservatoires et les écoles de musique franciliennes pour leur proposer d'ouvrir des cours de harpe. Sa démarche n'est toutefois que rarement couronnée de succès. En effet, le prix d'une harpe étant très élevé (un minimum de 2000 euros pour un maximum de 20000 ), peu d'institutions de formation musicale sont en mesure de procéder à un tel investissement.

Elle parvient à ses fins dans quelques conservatoires mais se heurte toujours au même problème. En participant à l'ouverture d'une classe de harpe, elle n'a, au départ, qu'un nombre limité d'élèves, qui plus est des élèves débutants à qui l'on donne seulement vingt minutes de cours hebdomadaires, ce qui ne lui permet pas d'accéder au volume horaire suffisant pour être à temps complet (16 heures). Elle doit donc à la fois s'arranger pour fidéliser ses élèves et en obtenir de nouveaux, mais aussi travailler simultanément dans plusieurs écoles de musique. Elle explique ainsi avoir été pendant un temps employée dans cinq conservatoires simultanément. Néanmoins, malgré tous ses efforts, ses enseignements en harpe ne suffisent pas à lui dégager un revenu suffisant. Pendant une vingtaine d'années à la suite de sa sortie du CNSM, elle est contrainte de donner des cours de solfège pour agrémenter son salaire: «Donc pour démarrer, ça a été une vraie difficulté, une très très grande difficulté. La vie a été très dure pour nous pendant vingt ans. Alors j'ai accepté de faire beaucoup de cours de formation musicale et de solfège pour avoir ce qu'il fallait pour vivre. Donc j'ai fait quinze ans professeur

34. Source: Ministère de la Culture et de la Communication/DEPS (2011), «Éducation artistique», Chiffres clés 2011. Statistiques de la culture, p. 201. 
de solfège et en parallèle je montais des classes de harpe jusqu'à ce que j'obtienne des classes pleines. Donc j'avais cinq postes dans la région parisienne. Chaque jour, j'allais dans une autre école. Et petit à petit, j'arrivais à me faire 2000 francs par mois, enfin ce qui était un minimum pour vivre à l'époque. C'est comme 1000 euros, même pas 1000 euros aujourd'hui. Donc ça a duré vingt ans.»

Cette situation professionnelle relativement précaire n'entraîne toutefois pas de renoncement ou de lassitude chez Christine. En effet, même si elle révèle les difficultés rencontrées pendant cette période, elle les envisage comme une étape qu'elle savait incontournable du fait de la pratique de la harpe. Fille et femme de musiciens, elle a ainsi toujours baigné dans le milieu de la musique classique et a donc, de tout temps, a fait face aux réalités objectives du métier, et notamment aux débouchés probables en fonction des instruments. Cette connaissance de l'univers musical lui a permis de ne pas fantasmer sur des possibles devenirs professionnels ou de ne pas se bercer d'illusions quant à sa carrière de harpiste ${ }^{35}$. Son engagement dans l'enseignement n'a donc pas été perçu comme un renoncement, mais plutôt comme une orientation logique compte tenu des postes disponibles dans les orchestres. De même, ce long moment d'instabilité professionnelle a pu être surmonté, car appréhendé comme la conséquence de la concurrence acharnée sur ce type d'emploi, et non comme un déficit de compétences. Cela d'autant plus que son environnement familial l'a soutenue dans cette situation d'attente autant moralement, en partageant son analyse des faits, que matériellement, en remédiant ponctuellement à ses difficultés financières. Les bénéfices de l'héritage musical sont d'ailleurs parfaitement intériorisés par Christine qui les souligne pour sa trajectoire et celles de ses filles: "On a un plus parce qu'on sait ce que c'est. Moi, mes filles, elles savent. Toutes les deux, elles sont entrées dans la musique, mais elles savent très bien que c'est dur. Elles savent que c'est dur mais elles le font. (...) Elles ont pas d'illusions, elles ont pas d'illusions. Elles le font parce qu'elles aiment ça. La seconde qui est dans une école là, elle est la seule à être fille de musiciens. Les autres viennent pour chercher un métier et ils comprennent pas que si on gagne déjà 1000 euros ou 1500 euros par mois, on va être heureux. Et il faut pas voir ça comme ça. C'est pas pour gagner sa vie. (...) Il faut être honnête, c'est pas rose tous les jours. Alors là, quand on a des familles, des parents musiciens, on est super gâté parce que les parents sont là en disant: "Pas de problème. Continue. On t'aide. Baisse pas les bras. On est là. T’y arriveras.” Ça, c'est génial, ça, c'est génial. Quand on n’a pas des parents musiciens, ils risquent de vous dire: "Eh bien pourquoi t'as fait ça?”»

Tous ces éléments sont alors à prendre en considération lorsque l'on analyse les «efforts» de Christine pour sortir de son statut précaire. Ne cédant pas au renoncement, elle continue de se produire ponctuellement en soliste lors de concerts. Elle obtient son CA de harpe, puis écrit une méthode d'enseignement de cet instrument, qui sera reprise dans les années 2000 au sein des programmes du

35. Une approche similaire est développée par Bernard Lehmann pour expliquer le parfait ajustement des «héritiers» au sein de l'orchestre symphonique (Lehmann, 2005). 
ministère de la Culture. Elle crée également, au début des années 1990, un stage estival où elle reçoit des élèves de tout niveau. Elle se présente à de nombreux concours de recrutement de professeur titulaire et déménage régulièrement au gré des différentes opportunités d'emploi qui lui sont proposées. À la fin des années 1990, elle est ainsi en Savoie avec toute sa famille où elle a monté une classe de harpe dans un conservatoire. C'est en 2000 que sa persévérance est récompensée. À 50 ans, elle est recrutée parmi une soixantaine de candidats pour un poste de professeur titulaire dans un CRR de province.

Pour obtenir ce dernier, elle a dû faire preuve de ses «qualités» d'instrumentiste mais aussi de pédagogue. Avec cet instrument pour lequel tous les postes sont soumis à une concurrence draconienne, et où l'accession à un poste pérenne est donc gage de réussite professionnelle, elle a réussi à se démarquer des autres prétendants par ses prestations instrumentales et son investissement dans l'activité pédagogique. Si elle se produit toujours en soliste, c'est dès lors à l'enseignement qu'est consacrée la majorité de son activité professionnelle. Elle maintient ses stages estivaux et se montre extrêmement attentive à la formation de ses élèves du CRR. Elle est d'ailleurs fière de pouvoir attester de la réussite professionnelle de plusieurs d'entre eux. Elle va même encore plus loin dans son investissement pédagogique en impulsant, en 2010, la création d'un concours de harpe destiné aux élèves de tous niveaux. Ce concours est immédiatement devenu le plus important organisé en France. Pour celui-ci, elle convoque des jurys de harpistes prestigieux et des élèves venant de l'Europe entière. Cette initiative lui a alors permis de «se faire un nom» dans l'univers de la harpe. Grâce au concours et à l'accumulation de capital social qui en découle, elle entretient maintenant des relations internationales et est amenée à se produire en soliste à l'étranger. Elle parvient ainsi à une forme de considération artistique grâce, en grande partie, à l'organisation de ce concours et donc à la pédagogie.

L'analyse croisée de ces deux trajectoires, qui traduit les inégalités instrumentales en révélant la difficulté de l'insertion professionnelle d'interprètes jouant d'instruments n'offrant que de faibles débouchés (que ce soit dans le secteur de l'enseignement et/ou le domaine de l'interprétation), souligne également les conditions de production du rapport à l'enseignement. Le ressentiment d'Yves quant à l'activité pédagogique doit se comprendre comme la congruence entre l'absence d'un héritage musical professionnel, le décalage produit par l'engagement d'un enfant des classes supérieures dans un instrument davantage joué par les classes moyennes - et qui a des perspectives d'emploi restreintes - et la relative facilité d'accès aux postes d'enseignant de saxophone. Ces éléments le conduisent à des aspirations initiales non ajustées à l'état du marché du travail pour saxophoniste et à une perception de la pédagogie comme relégation musicale. À l'inverse, pour Christine, issue d'un milieu musical et initiée précocement à la limitation des devenirs professionnels possibles à la harpe, l'accès au poste pérenne et à temps complet d'enseignant n'est pas un renoncement mais représente une forme de réussite professionnelle, qui symbolise autant ses qualités instrumentales et pédagogiques que son obstination, et donc sa vocation. 
De ces rapports contradictoires à la pratique pédagogique, et donc à l'occupation d'une position intermédiaire, découlent deux positionnements opposés vis-à-vis des formes routinisées de la reconnaissance musicale. Le premier, représenté par Yves, ne s'inscrit pas dans une remise en cause du modèle traditionnel mais pointe davantage les inégalités instrumentales d'accès aux positions dominantes — sans toutefois les réinterpréter comme des inégalités sociales. La légitimité de la «forme traditionnelle» de consécration n'est pas déniée, mais au contraire jalousée par cet instrumentiste formé selon cet «idéal» et qui ne peut, en raison de la structure du marché du travail musical, y prétendre. Il s'agit alors de tenter d'atteindre — en vain — la consécration avec le recours à la pluri-activité dans une sorte de mimétisme avec le mode traditionnel de reconnaissance, tout en considérant l'enseignement comme une activité alimentaire. À l'inverse, le second positionnement, caractérisé par Christine, propose une réinterprétation originale. Préparée par son environnement familial à «faire de nécessité vertu», elle ne ressent pas la pratique pédagogique comme une contrainte. Au contraire, elle parvient même à réorienter sa quête de reconnaissance autour de l'activité pédagogique en en faisant la base de son processus de valorisation artistique.

\section{CONCLUSION}

Le processus de consécration musicale, sous sa forme routinisée et s'appliquant au groupe des instrumentistes classiques français, ne saurait se réduire à la révélation d'un «talent» qui, par un procédé quasi mécaniste, engendrerait la reconnaissance artistique du musicien à qui on l'attribue. Étudier ce processus nécessite de dépasser les considérations sur les qualités individuelles qui n'ont de valeur qu'inscrites dans un espace socialement construit. Plus que le «talent», ce sont les conditions sociales de sa reconnaissance et de son objectivation, déterminées par la structure du champ investi, qui fondent l'accès à la reconnaissance artistique. La consécration musicale s'insère ainsi dans l'espace des possibles instrumentaux, qui, en offrant aux instruments les plus socialement sélectifs des possibilités accrues d'accès aux positions dominantes, consacre autant les qualités musicales que sociales.

Pour autant, si la notion de «talent» est fortement amendée par l'objectivation sociologique, elle demeure une référence fondamentale pour les instrumentistes percevant leur métier comme la concrétisation d'une vocation. «Parce qu'il appartient au travail d'inculcation de la vocation d'imposer, en même temps que la vocation, la méconnaissance des déterminismes qui la rendent possible» (Suaud, 1978: 10), les musiciens classiques demeurent habités d'un sentiment de prédestination et convaincus de la centralité du don dans les classements artistiques, croyances entretenues autant par la sphère familiale (Augustins, 1991) que le système de formation (Morinière, 2007). En ce sens, le fait de ne pas accéder aux positions dominantes, de ne pas atteindre la consécration routinisée, même s'il relève des modes de structuration du champ, peut être destructeur pour l'interprète puisque cette nonconsécration remet en question sa croyance dans l'existence de son «talent». Reste alors à se « rassurer» par des voies détournées liées à la trajectoire sociale et profes- 
sionnelle des musiciens, qui peuvent être, comme pour Yves, une activité de concert annexe à l'enseignement ou, pour Christine, une revalorisation artistique de la pratique pédagogique.

La construction sociale de l'espace produit ainsi des effets qui ne se limitent pas aux principes de consécration mais se retrouvent également lorsqu'il s'agit de surmonter une absence de reconnaissance par l'adoption de stratégies de réassurance. De ce fait, l'utilisation de la notion de trajectoire, parce qu'elle permet de faire cohabiter différentes «histoires sectorielles» (familiales, scolaires, musicales, etc.) dans une approche biographique (Mauger, Poliak et Pudal, 1999), qu'elle rétablit l'influence des facteurs sociaux dans la réussite musicale et qu'elle prend en compte la structure du champ dans la dynamique des mouvements (Bourdieu, 1986), apparaît comme le concept le plus approprié pour retraduire les modalités du cheminement professionnel des instrumentistes dans un champ de la musique classique à l'autonomie relative.

\section{RÉSUMÉ}

Cet article analyse les déterminants sociaux de l'accès à la reconnaissance artistique en musique classique. Récusant les modèles théoriques naturalistes centrés sur le talent comme principe explicatif ultime de la réussite des carrières artistiques, il s'attache à mettre en évidence les conditions sociales de la consécration musicale. Ainsi, le processus de reconnaissance est étudié à travers les inégalités instrumentales qu'il suscite, lesquelles sont étroitement liées à des différenciations sociales. Après avoir mis au jour ces inégalités, qui tendent à consacrer prioritairement des interprètes d'instruments socialement sélectifs, on s'intéresse aux façons de surmonter l'absence de consécration pour des interprètes des instruments plus ouverts socialement qui sont, le plus souvent, poussés vers l'enseignement. L'étude des trajectoires de musiciens révèle ainsi les dynamiques sociales auxquelles elles sont soumises, de l'origine sociale dont dépend notamment la familiarisation précoce avec les principes du champ de la musique classique aux inégalités entre les sexes qui orientent les manières de «se faire à sa position».

Mots clés: musique, consécration artistique, division sociale du travail, inégalités instrumentales, revalorisation de l'enseignement

\section{ABSTRACT}

This paper analyses the social determinants of access to artistic recognition in classical music. Rejecting naturalist theoretical models focused on talent as the ultimate explanatory principle of the success of artistic careers, it aims to highlight the social conditions of acclaim in music. Thus, the recognition process is studied through the instrument inequalities it creates, which are closely tied to social differentiations. After revealing these inequalities, which tend to lead mainly to the recognition of interpreters of socially selected instruments, the author examines ways used by to interpreters of instruments that are socially more open to overcome the lack of recognition they receive. More often than not, these musicians are encouraged to teach. The study of musicians' career paths reveals the social dynamics they are subjected to, from social origin, which conditions in particular their early familiarization with the principles of classical music, to gender inequalities that shape the way in which they "accept their status." 
Key words: music, artist recognition, social division of labour, instrument inequalities, revaluation of teaching.

\section{RESUMEN}

Este artículo analiza las determinantes sociales del acceso al reconocimiento artístico en música clásica. Desafiando los modelos teóricos naturalistas centrados en el talento como principio explicativo último del éxito de las carreras artísticas, el artículo busca evidenciar las condiciones sociales de la consagración musical. Así, el proceso de reconocimiento es estudiado a través de las desigualdades instrumentales que éste suscita, las cuales se encuentran estrechamente asociadas a diferenciaciones sociales. Después de evidenciar estas desigualdades que tienden a consagrar prioritariamente a intérpretes de instrumentos socialmente selectivos, el artículo se interesa en las maneras de superar la ausencia de consagración en aquellos intérpretes de instrumentos más abiertos socialmente que son, con mayor frecuencia, empujados hacia la enseñanza. El estudio de las trayectorias de los músicos revela así las dinámicas sociales a las cuales aquellas son sometidas, del origen social del cual depende particularmente la familiarización precoz con los principios del campo de la música clásica, a las desigualdades entre sexos que orientan las maneras de "hacerse a esta posición".

Palabras clave: música, consagración artística, división social del trabajo, desigualdades instrumentales, revalorización de la enseñanza.

\section{BIBLIOGRAPHIE}

Adenot, P. (2008), Les musiciens d'orchestre symphonique: de la vocation au désenchantement, Paris, L'Harmattan, coll. «Univers musical».

Augustins, G. (1991), «Le don chez les musiciens», in Segalen, M. (dir.), Jeux de famille, Paris, Presses du CNRS, p. 99-113.

Balland, L. (2012), «Un cas d'école. L'entrée dans le métier de professeur d'une "enfant de la démocratisation scolaire" ", Actes de la recherche en sciences sociales, n 191-192, p. 40-47.

Bourdieu, P. (1975), «L'invention de la vie d'artiste», Actes de la recherche en sciences sociales, nº 2, p. 67-93.

Bourdieu, P. (1982), «Les rites comme actes d'institution», Actes de la recherche en sciences sociales, $\mathrm{n}^{\circ} 43$, p. 58-63.

Bourdieu, P. (1986), «L'illusion biographique», Actes de la recherche en sciences sociales, nº 62-63, 1986, p. 69-72.

Bourdieu, P. (2013), Manet. Une révolution symbolique, Paris, Seuil/Raisons d'agir.

Coulangeon, P. (1999), «Les mondes de l'art à l'épreuve du salariat. Le cas des musiciens de jazz français», revue française de sociologie, vol. 40, $\mathrm{n}^{\circ} 4$, p. 689-713.

Coulangeon, P. (2004), Les musiciens interprètes en France. Portrait d'une profession, Paris, La documentation Française, ministère de la Culture et de la Communication, Département des études et de la prospective.

Coulangeon, P. et Ravet, H. (2003), «La division sexuelle du travail chez les musiciens français», Sociologie du travail, vol. $45, \mathrm{n}^{\circ} 3$, p. 361-384.

Cueille, C. (2004), «Écoles de musique et espaces territoriaux: une relation sous tutelle», Sociologie des faits musicaux et modèles culturels, Observatoire Musical Français, $\mathrm{n}^{\circ}$ 10, p. 3-28.

DeNora, T. (1995), «Beethoven et l'invention du génie», Actes de la recherche en sciences sociales, n 110 , p. 36-45.

Duвors, V. (1999), La politique culturelle. Genèse d'une catégorie d'intervention publique, Paris, Belin, coll. «Socio-Histoires». 
Dubors, V. (2013), La culture comme vocation, Paris, Raisons d'agir, coll. «Cours et travaux».

Dubois, V., Méon, J.-M. et Pierru E. (2009), Les mondes de l'harmonie. Enquête sur une pratique musicale amateur, Paris, Éd. La Dispute.

Durkheim, E. (1988), Les règles de la méthode sociologique, Paris, Flammarion, coll. «Champs», [1895].

Euias, N. (1991), Mozart, sociologie d’un génie, Paris, Le Seuil, coll. «La librairie du xxe siècle».

Escal, F. et Rousseau-Dujardin, J. (1999), Musique et différence des sexes, Paris, L'Harmattan.

Fleuriel, S. (2004), «De la vocation artistique à la précarité: devenir professeur de danse jazz», Sociologie de l'art, $\mathrm{n}^{\circ} 4$, p. 137-157.

Ganvert, G. (1999), L'enseignement de la musique en France. Situations, problèmes, réflexion, Paris, L'Harmattan, coll. «Sciences de l'éducation musicale».

Gerbod, P. (1980), «L'institution orphéonique en France du $\mathrm{XIX}^{\mathrm{e}}$ au $\mathrm{Xx}^{\mathrm{e}}$ siècle», Ethnologie Française, vol. $10, \mathrm{n}^{\circ} 1, \mathrm{p} .27-44$.

Godechot, O. (2010), «Pierre-Michel Menger, Le travail créateur. S’accomplir dans l'incertain», Sociologie, vol. $1, \mathrm{n}^{\circ} 1$.

Gumplowicz, P. (2001 [1987]), Les travaux d'Orphée. Deux siècles de pratique musicale en France (18202000), Paris, Aubier.

Lahire, B. (1995), Tableaux de famille. Heurs et malheurs scolaires en milieux populaires, Paris, Gallimard, coll. «Hautes Études».

Lehmann, B. (2005), L'orchestre dans tous ses éclats. Ethnographie des formations symphoniques, Paris, La Découverte, coll. «Sciences humaines et sociales».

Lenoir, R. (1979), "Notes pour une histoire sociale du piano», Actes de la recherche en sciences sociales, $\mathrm{n}^{\circ} 28$, p. $79-82$.

Leterrier, S.-A. (1999), «Musique populaire et musique savante au XIX siècle. Du "peuple" au "public" ", Revue d'histoire du XIX $x^{e}$ siècle, ${ }^{\circ} 19$, p. 89-103.

Lizé, W., Naudier, D. et Roueff, O. (2011), Intermédiaires du travail artistique. À la frontière de l'art et du commerce, Paris, La documentation Française.

Mauger G., Poliak C. et Pudal B., Histoires de lecteurs, Paris, Nathan, 1999.

Mauger, G. (2006), "Le capital spécifique», in MAUger, G. (dir.), L'accès à la vie d'artiste. Sélection et consécration artistiques, Bellecombe-en-Bauges, Le Croquant, p. 237-253.

Menger, P.-M. (2001), Le paradoxe du musicien. Le compositeur, le mélomane et l'État dans la société contemporaine, Paris, l'Harmattan, coll. «Logiques sociales».

Menger, P.-M. (2002a), «Le génie et sa sociologie. Controverses interprétatives sur le cas de Beethoven», Annales. Histoire, Sciences sociales, vol. 57, nº 4, p. 967-999.

Menger, P.-M. (2002b), Portrait de l'artiste en travailleur. Métamorphoses du capitalisme, Paris, Le Seuil, coll. «La République des idées».

Menger, P.-M. (2009), Le travail créateur. S’accomplir dans l’incertain, Paris, Gallimard-Seuil, coll. «Hautes Études».

Menger, P.-M. (2010), «À quelles conditions peut-on créer? Entretien avec Pierre François», Critique, $\mathrm{n}^{\circ} 761$, p. $852-864$.

Merton, R. K. (1968), «The Matthew Effect in Science», Science, vol. 159, n 3810, p. 56-63.

Monnot, C. (2012), De la harpe au trombone. Apprentissage instrumental et construction du genre, Rennes, PUR, coll. «Le sens social».

Morinière, T. (2007), «Les petites voies de la chanson de variétés. La révélation du charisme du chant et la démocratisation de la vocation d'interprète", Actes de la recherche en sciences sociales, $n^{\circ} 168$, p. 68-81.

Pégourdie, A. (2012), «Effets de génération dans le rapport au métier des intermittents en musique classique», in Chantegros S., Orange S., Pégourdie A. et Rougier C. (dir.), La fabrique biographique, Limoges, Pulim, coll. «Sociologies et Sciences sociales», p. 145-161.

PÉGourdie, A. (2013), Les provinces de la musique. Pratiques professionnelles, trajectoires et rapport au métier des instrumentistes classiques limougeauds, Limoges, Université de Limoges, Thèse de doctorat de sociologie. 
PÉgourdie, A. (2015), «L' «instrumentalisation» des carrières musicales. Division sociale du travail, inégalités d'accès à l'emploi et renversement de la hiérarchie musicale dans les conservatoires de musique», Sociologie, vol. 6, $n^{\circ}$ 4, p. 321-337.

Ravet, H. (2003), «Professionnalisation féminine et féminisation d'une profession: les artistes interprètes de musique», Travail, genre et sociétés, $\mathrm{n}^{\circ}$ 9, p. 173-195.

RAvet, H. (2006), «L'accès des femmes aux professions artistiques. Un double droit d'entrée dans le champ musical», in Mauger, G. (dir.), L'accès à la vie d'artiste. Sélection et consécration artistiques, Bellecombe-en-Bauges, Le Croquant, p. 161-176.

Rosen, S. (1981), «The Economics of Superstars», The American Economic Review, vol. 71, n 5, p. 845-858.

SAPIro, G. (2007), «La vocation artistique entre don et don de soi», Actes de la recherche en sciences sociales, $\mathrm{n}^{\circ} 168$, p. $4-11$.

Sснотте́, M. (2012), La construction du «talent». Sociologie de la domination des coureurs marocains, Paris, Raisons d'agir, coll. «Cours et travaux».

Sснотте, M. (2013), «Le don, le génie et le talent. Critique de l'approche de Pierre-Michel Menger», Genèses, nº 93, p. 144-164.

Sorignet, P.-E. (2004a), «Un processus de recrutement sur un marché du travail artistique: le cas de l'audition de danse contemporaine», Genèses, n 57 , p. 64-88.

Sorignet, P.-E. (2004b), «Sortir d'un métier de vocation: le cas des danseurs contemporains», Sociétés contemporaines, $\mathrm{n}^{\circ}$ 56, p. 111-132.

SuAud, C. (1978), La vocation. Conversion et reconversion des prêtres ruraux, Paris, Minuit, coll. «Le sens commun».

Teillet, P. (2003), «Publics et politiques des musiques actuelles», in Donnat, O. et Tolila, P. (dir.), $L e(s)$ public(s) de la culture, Paris, Presses de Sciences Po, p. 155-180.

WAGNER, I. (2004), «La formation des violonistes virtuoses: les réseaux de soutien », Sociétés contemporaines, $\mathrm{n}^{\circ} 56$, p. $133-163$.

Weber, M. (1995), Économie et société. Tome 1. Les catégories de la sociologie, Paris, Plon, coll. «Agora les classiques", [1922]. 